International Journal of Engineering \& Technology, $7(1.2)(2018) 171-174$
SPC
International Journal of Engineering \& Technology
Website www.sciencepubco.com/index.php/IJET
Research paper

\title{
Comparative study of cluster based routing protocols in WSN
}

\author{
Varsha Bhatia $^{1 *}$, Sunita Kumawat ${ }^{1}$, Vivek Jaglan ${ }^{1}$ \\ ${ }^{1}$ Department of Computer Science \& Engineering, Amity University Haryana, India \\ *Corresponding author E-mail: varsha.bhatia.in@ gmail.com
}

\begin{abstract}
Wireless Sensors network is a type of wireless network, used in diverse applications and has its own set of challenges. Apart from organizing and managing WSN, the main challenges include limited resources, dynamic topology and low scalability. Wireless Sensor nodes are battery operated, so energy scarceness is a major concern. The energy consumption is maximal at the time of data transmission between network devices or nodes. Various energy conservation schemes are applied in WSN; Energy Efficient Routing is one of the possible solutions. Energy Efficient Routing is used to minimize the maintenance cost of the network and maximize the performance of the node. In this paper different hierarchical cluster based routing protocols are discussed.
\end{abstract}

Keywords: Wireless Sensor Network; Clustering; Routing Protocols.

\section{Introduction}

Routing is a procedure of finding way or courses to dependably convey information parcels from source to goal. If there should be an occurrence of death of couple of hubs, directing calculation is in charge of keeping up or repairing broken courses or framing new courses between hubs. Steering conventions can either be Data Centric, Hierarchical or Location based. Information Centric conventions are inquiry construct and work with respect to the premise of naming the coveted information. Various leveled directing gatherings sensor hubs to shape bunch, chooses a group head, which totals and expels copy information (got from the hubs exhibits in bunch) and afterward transmit it to sink. Area construct steering depend in light of area to transfer information to its goal. In this work, different Hierarchical group based directing conventions are talked about. Various leveled bunch based steering convention, offer adaptability, vitality proficiency and enhanced system lifetime.

\section{Clustering}

Clustering is a practice used to extend the network lifetime of sensor network by conserving energy.WSN deploys nodes in hundreds and thousands, either in structural way or randomly. Cluster based protocols mostly group the wireless sensor nodes into clusters and provide various advantages to the WSN such as evenly distributing the energy, reducing overhead, reducing data redundancy and effectively organizing the network. Clustering algorithm perform grouping of nodes, based on certain norm. Cluster Head $(\mathrm{CH})$ for each cluster is selected. Each node sense its data performs computations, and transmit it to $\mathrm{CH}$. The role of cluster head is to aggregate the data received from its members, and to act as decision maker in various routing and scheduling tasks. Data aggregation reduces the energy consumption in sensor node by reducing message overhead and improving the resource utilization.
In clustering algorithm cluster head is selected, with the major design objective to extend the network lifetime, and to minimize the energy consumption. $\mathrm{CH}$ performs more complex task than ordinary sensor nodes, hence consumes more energy. An improper selection of $\mathrm{CH}$ can lead to communication interference and network partitioning; this normally occurs if the distance between $\mathrm{CHs}$ and Base station (BS) is large. A proper selection of $\mathrm{CH}$ and its role rotation ensures uniform load balancing and energy consumption.

\subsection{Clustering process}

i). Cluster Formation Phase: In cluster formation phase, nodes are divided in groups or clusters. Generally cluster parameters required for $\mathrm{CH}$ selection are evaluated and are transmitted to the neighbors' (in distributed approach) or to the base station (BS) through $\mathrm{CH}$ (in centralized approach).In centralized approach; BS selects the cluster head and informs the decision to the nodes. Hence BS requires global knowledge of the network. In distributed approach, each sensor node has to decide on its own, which cluster to join.

ii). Cluster Head Selection Phase: Each cluster selects a sensor node as its $\mathrm{CH}$ based on some criteria. The choice of criteria depends upon the objectives of Clustering. Generally clustering algorithms are multi objective in nature. Few of these objectives are Energy efficiency, network lifetime, load balancing, guarantee of connectivity \& quality of service. In homogenous environment, all sensor nodes are equally capable in terms of energy, communication and computational power; in this case $\mathrm{CH}$ selection is based on certain criteria. In heterogeneous environment, nodes have unequal capabilities and it is desirable to choose more powerful node as CHs. 


\section{Comparison of cluster based routing proto- cols}

\subsection{Low energy adaptive clustering hierarchy (LEACH)}

LEACH [2] was composed with the plan to pivot the part of bunch head and guarantee that same hub isn't chosen as group head in consequent interim. The calculation is appropriated in nature; hubs are permitted to take autonomous choices, with no focal control $\mathrm{CH}$ hub plays out the part of information aggregator and transmits the collected message to BS.

The operation in LEACH is partitioning into rounds. A round comprises of two stages' setup stage and consistent stage. Amid setup stage groups are framed and bunch head are chosen. In consistent detected information is transmitted to BS through CHs. Drain is probabilistic bunching calculation; every hub figures its likelihood of getting to be $\mathrm{CH}$, by creating an irregular number in the vicinity of 0 and 1 . Hubs with likelihood lower than an edge esteem are chosen as $\mathrm{CH}$.

$\mathrm{T}(\mathrm{n})=\left\{\begin{array}{c}\frac{\mathrm{P}}{1-\mathrm{P}\left(\mathrm{r} \bmod \frac{1}{\mathrm{p}}\right)} \text { if } \mathrm{n} \in \mathrm{G} \\ 0 \text { otherwise }\end{array}\right\}$

The above equation is used to calculate the threshold value for the round $\mathrm{r}, \mathrm{P}$ is the desired percentage of $\mathrm{CH}$ required for the network and $\mathrm{G}$ is set of nodes, which have not been selected as $\mathrm{CH}$ in last $1 / \mathrm{P}$ rounds.

Each sensor node gets opportunity to become $\mathrm{CH}$, the final decision depends upon the suggested percentage $\mathrm{P}$ number of cluster heads for the network. All selected $\mathrm{CHs}$ broadcast advertisement message, each non $\mathrm{CH}$ node joins the $\mathrm{CH}$ based on received signal strength of $\mathrm{CH}$ advertisement signals. Each $\mathrm{CH}$ transmits TDMA schedule to its member nodes, and member nodes transmit their sensed data in their respective slots. Each node can turn on the radio, only in their time slot and keep it off for rest of the steady phase duration. After a pre determined time, the network goes back into the set-up phase again and enters another round of $\mathrm{CH}$ election.

LEACH is entirely distributed; all decisions are taken locally \& independently without any intervention from the BS. Cluster heads may not be well separated and uniform (by probability mechanism), and number of cluster heads formed vary in each round.

\subsection{Hybrid energy efficient distributed clustering (HEED) [3]}

HEED is a distributed clustering algorithm; it uses residual energy as main parameter for clustering. HEED [3] is different from LEACH; as it uses multi hop inter cluster communications. $\mathrm{CH}$ selection is based on two criteria. First one being residual energy, second one is the intra cluster communication cost Communication cost comes into play when there is tie between two $\mathrm{CH}$ candidates having same residual energy. HEED follows a hybrid approach, $\mathrm{CH}$ are selected probabilistically based on residual energy and sensor node join a $\mathrm{CH}$, so that communication cost is minimized.

Heed uses a predefined value for $\mathrm{C}$ prob, which represents the percentage of $\mathrm{CHs}$ in the network. The probability of sensor node to become $\mathrm{CH}$ is calculated using the formula.

$\mathrm{CH}_{\text {prob }}=\mathrm{C}_{\text {prob }} \frac{\text { Eres }}{\text { Emax }}$

Generally CHprob is not permissible to drop below a threshold value, this is inverse of maximum reference energy of the node $\mathrm{E}$ max. The Eres represents expected residual energy of the node. Each node has to go through a number of iterations, until it finds its $\mathrm{CH}$. Each node doubles value of its $\mathrm{CHprob}$ after each iteration, until its $\mathrm{CHprob}$ reaches one. Each node is assigned a tentative $\mathrm{CH}$ status, if it's $\mathrm{CHprob}$ less than one. If $\mathrm{CHprob}$ of a node is equal to 1 , it becomes permanent $\mathrm{CH}$. Both $\mathrm{CH}$ candidates (tentative and permanent) announce their status to neighboring nodes. A tentative $\mathrm{CH}$ status will change to regular node afterwards, if it hears from a $\mathrm{CH}$ with lower communication cost than itself. A node announces itself as $\mathrm{CH}$, if it doesn't hear from any other node. A node can become $\mathrm{CH}$ in successive intervals, if residual energy is high and cost is minimal.

HEED algorithm terminate after finite number of iterations, independent of cluster size. HEED does not make any assumptions about the density of nodes, or about node capabilities, locationawareness etc. The process of clustering is independent of the network topology and terminates in $\mathrm{O}(1)$ iterations.

\subsection{Threshold sensitive energy efficient sensor network protocol (TEEN) [4]}

TEEN was proposed to deal with abrupt changes in sensed value such as temperature. It is suitable for reactive scenes. It requires two thresholds for its operations, which depends on the range \& tolerance limit of the attribute to be sensed, for the specific application. These thresholds are termed as hard threshold and soft threshold. Sensor node senses the environment continuously, but transmits less frequently. Hard threshold allows node to transmit, only when data matches the range of interest. Soft threshold is similar to the tolerance limit of the sensed attribute. The transmission of data is not allowed, if it is below the soft threshold value. The value of thresholds is transmitted, at every cluster head change time. Threshold values can be varied, as per requirement. Information propagation happens only through $\mathrm{CH}$, hence it is required that $\mathrm{CH}$ are in communication range of its neighboring CHs. TEEN is a useful protocol for Time critical application but it is not suitable for periodic data gathering. It is difficult to identify dead nodes, as sensors don't report regularly.

\subsection{Adaptive periodic threshold sensitive energy effi- cient sensor network protocol (APTEEN) [5]}

APTEEN is an enhanced version of TEEN, and is suitable both for periodic data gathering and event driven. It can be used for both proactive and reactive policies. Algorithm is query based and three types of queries are permissible. Historic query which allow extracting information from the past records. One time query to take snapshot of the current situation. Persistent query to observe environment for a time interval. In APTEEN the $\mathrm{CH}$ selection method is similar to LEACH-C. CH broadcast four attributes to its member node. The first being the sensor data or attribute based on WSN application. Like TEEN, it transmits hard threshold (HT) and soft threshold (ST) values. TDMA schedule through which it assigns time slots for each member node. The last attribute is the Count time (CT), which is the maximum permissible interval, between two consecutive transmissions by a node. Count time is the time, generally multiple of TDMA schedule length. Count time (CT) accounts for proactive part, if a node has not transmitted any data for CT time interval it is forced to sense and transmit the data to $\mathrm{CH}$. Users have the flexibility to change both threshold and count time. The Cluster can exist for a definite time called cluster period, after which Base Station (BS) reorganizes the network.

\subsection{Two-level hierarchy LEACH (TL LEACH) [6]}

TL- LEACH $[1,6]$ is an extended form of LEACH. In TLLEACH CH collects data from its member nodes and send it to Base station in two hops. TL- LEACH maintains a two level hierarchy of Cluster Heads. First layer consists of primary CHs, which act as a relay node to transmit data to Base station. Second layer comprises of secondary $\mathrm{CHs}$ and normal node. The role of Secondary $\mathrm{CHs}$ is to collect the data from normal node, aggregate and transmit it to respective primary $\mathrm{CH}$.

The method of $\mathrm{CH}$ selection is same as in $\mathrm{LEACH}$, each node decides upon its role to act as primary $\mathrm{CH}$, secondary $\mathrm{CH}$ or 
normal node. The prior probability of primary $\mathrm{CH}$ is less than that of Secondary $\mathrm{CHs}$. Both primary $\mathrm{CHs}$ and secondary $\mathrm{CHs}$ send advertisement message to neighboring nodes through Code Division Multiple Access (CDMA) codes. Primary $\mathrm{CH}$ creates TDMA schedule for its Secondary $\mathrm{CHs}$ and normal nodes, secondary $\mathrm{CH}$ transmit this schedule to its normal nodes. Clusters are formed and each normal node transmits the data according to its TDMA slot.

\subsection{Energy efficient unequal clustering EEUC [7]}

EEUC is a distributed clustering algorithm, which selects $\mathrm{CH}$ based on local competition. Each sensor node has a pre assigned competitive radius; that depends on its distance from the Base Station. The greater the distance from the BS, larger will be its competition radius. Competitive radius [1] can be calculated using the formula given below

$$
\mathrm{R}(\mathrm{Si})=\left(1-\mathrm{c} \frac{\mathrm{dmax}-\mathrm{d}(\mathrm{Si}, \mathrm{BS})}{\mathrm{dmax}-\mathrm{dmin},}\right) \mathrm{Rcomp}
$$

Where Rcomp is pre characterized most extreme permitted rivalry range, dmax is the greatest separation of hub from the BS, dmin is the base separation of hub from the BS and $\mathrm{d}(\mathrm{Si}, \mathrm{BS})$ is the separation between hub Si and BS. C is consistent, whose esteem lies in the vicinity of 0 and 1 . EEUC frame groups of unequal size, bunch close BS are littler in measure, and more distant ones are expansive in estimate. EEUC utilizes multi jump steering for entomb bunch correspondences; thus hubs close BS will go about as transfer hub and exhaust more vitality. On the off chance that a hub is near BS, little bunch estimate guarantees that intra group messages expends less vitality, thus more vitality is accessible for bury bunch correspondences. Consequently EEUC diminishes hotspot issue. Message overhead is high, as every hub partakes for $\mathrm{CH}$ choice in each round.

\subsection{Energy efficient clustering scheme (EECS)}

EECS similar to LEACH, but has better cluster formation and cluster Head selection Phase. EECS is a periodic data gathering algorithm. Residual energy is considered as a parameter for $\mathrm{CH}$ selection. A Cost function is used by non $\mathrm{CH}$ nodes to decide on which cluster to join. Cost function is a weighted function of distance between sensor node and $\mathrm{CH}$, and distance between $\mathrm{CH}$ and BS. Single hop communication is performed from sensor node to $\mathrm{CH}$ and to the $\mathrm{BS}$.

In deployment phase, a BS broadcast a beacon message, each node receives this beacon message and based on received signal strength calculates its distance from the base station. Based on this, sensor node chooses an appropriate power level for further communication. Each sensor node becomes a cluster head candidate with a probability value of $\mathrm{T}$ and sends out a COMPETE_MESSAGE advertising its ID and its residual energy value. The sensor nodes have to wait for a TIMER period within which they decide as to which cluster they want to join. If a sensor node hears another (Candidate) sensor node (within its Transmission Range) having higher residual energy then it joins that cluster as cluster member. Otherwise, it elects itself as a cluster head. In tie situations, sensor node ID is used to decide which of the sensor nodes would become a cluster head.

All the selected $\mathrm{CH}$ nodes also send their distance from BS in advertisement message. Each non-cluster head sensor node then calculates the cost metric of joining adjacent clusters. Each node chooses the $\mathrm{CH}$ for which the cost metric is minimal. Cost function for node $\mathrm{Pi}$ and $\mathrm{CHj}$ is given by

$\operatorname{cost}(\mathrm{j}, \mathrm{i})=\mathrm{w} * \mathrm{f}(\operatorname{dis}(\mathrm{Pi}, \mathrm{CHj})+(1-\mathrm{w}) * \mathrm{~g}(\operatorname{dis}(\mathrm{CHj}, \mathrm{BS}))$

The distribution of CHs in EECS is more uniform as compared to LEACH. Major drawback of EECS all nodes compete in each round for $\mathrm{CH}$ selection process, hence control message overheads are more than LEACH

\section{Comparison of cluster based routing proto- cols in WSN}

Table 1: Clustering Attributes of Routing Protocols in WSN

\begin{tabular}{|c|c|c|c|c|c|c|c|}
\hline PROTOCOL & LEACH & HEED & TL LEACH & EECS & EEUC & TEEN & APTEEN \\
\hline Cluster count & Variable & Variable & Variable & Variable & Variable & Fixed & Variable \\
\hline Cluster size & Even & Even & Even & Uneven & Uneven & Even & Even \\
\hline Classification & Probabilistic & Iterative & Probabilistic & Probabilistic & Probabilistic & Probabilistic & Probabilistic \\
\hline $\mathrm{CH}$ Selection & $\begin{array}{l}\text { Threshold } \\
\text { based } \\
\text { Probability }\end{array}$ & $\begin{array}{l}\text { Residual } \\
\text { Energy }\end{array}$ & $\begin{array}{l}\text { Threshold based } \\
\text { probability }\end{array}$ & $\begin{array}{l}\text { Residual } \\
\text { Energy }\end{array}$ & $\begin{array}{l}\text { Threshold } \\
\text { based } \\
\text { Probability }\end{array}$ & $\begin{array}{l}\text { Threshold } \\
\text { based } \\
\text { probability }\end{array}$ & $\begin{array}{l}\text { Threshold based } \\
\text { probability }\end{array}$ \\
\hline $\begin{array}{l}\text { Cluster formation } \\
\text { (which cluster to } \\
\text { join) }\end{array}$ & $\begin{array}{l}\text { Based on } \\
\text { Received } \\
\text { Signal } \\
\text { Strength(RSSI) }\end{array}$ & $\begin{array}{l}\text { Intra-Cluster } \\
\text { Communication } \\
\text { Cost }\end{array}$ & Based on RSSI & $\begin{array}{l}\text { Distance from } \\
\mathrm{CH} \text {, distance } \\
\text { of } \mathrm{CH} \text { from } \\
\mathrm{BS}\end{array}$ & $\begin{array}{l}\text { Competition } \\
\text { Radius, Based } \\
\text { on distance } \\
\text { from BS }\end{array}$ & $\begin{array}{l}\text { Based on } \\
\text { received } \\
\text { signal } \\
\text { strength }\end{array}$ & $\begin{array}{l}\text { Based on re- } \\
\text { ceived signal } \\
\text { strength }\end{array}$ \\
\hline Node Type & Homogenous & Homogenous & Homogenous & Homogenous & Homogenous & Homogenous & Homogenous \\
\hline Mobility & Stationary & Stationary & Stationary & Stationary & Stationary & Stationary & Stationary \\
\hline Intra cluster Routing & Single Hop & Single Hop & Single Hop & Single Hop & Single Hop & Single Hop & Single Hop \\
\hline Inter cluster routing & Single Hop & $\begin{array}{l}\text { Single/Multi } \\
\text { Hop }\end{array}$ & Multi Hop & Single Hop & Multi hop & Multi hop & Multi hop \\
\hline Control Manner & Distributed & Distributed & Distributed & Distributed & Distributed & Distributed & Distributed \\
\hline Cluster Parameters & Adaptive & Adaptive & Adaptive & Adaptive & Adaptive & Adaptive & Adaptive \\
\hline
\end{tabular}


Table 2: Advantages and Disadvantages of Clustering Protocols

\begin{tabular}{|c|c|c|}
\hline Protocol & Advantages & Disadvantages \\
\hline $\begin{array}{l}\text { LEACH } \\
{[2]}\end{array}$ & $\begin{array}{l}\text { - } \quad \text { Completely distributed protocol. } \\
\text { location information about node is not required for } \mathrm{CH} \\
\text { selection } \\
\text { - } \quad \text { Global knowledge about the network is not needed. }\end{array}$ & $\begin{array}{l}\text { - } \quad \text { LEACH assumes nodes to be Homogeneous which is unreal as } \\
\text { usually heterogeneity in energy exist. } \\
\text { - } \quad \text { Energy was not considered for } \mathrm{CH} \text { selection } \\
\text { - } \quad \text { Uneven distribution of } \mathrm{CH} \text { across the network } \\
\text { - } \quad \text { Not suitable for large networks }\end{array}$ \\
\hline TEEN [4] & $\begin{array}{l}\text { Choosing appropriate values of hard and soft threshold } \\
\text { can help to manage the tradeoff in number of Packet } \\
\text { transmissions \& Energy consumption. } \\
\text { Reliable data delivery } \\
\text { - } \quad \text { Suitable for event driven application. }\end{array}$ & $\begin{array}{l}\text { - Not suitable for continuous monitoring applications. } \\
\text { - } \quad \text { No communication will occur, if the desired thresholds are not } \\
\text { achieved, it creates an ambiguity whether the node is dead or } \\
\text { alive. }\end{array}$ \\
\hline $\begin{array}{l}\text { APTEEN } \\
{[5]}\end{array}$ & $\begin{array}{l}\text { Hybrid clustering protocol, suitable for both event driven } \\
\text { and periodic data collection application. }\end{array}$ & $\begin{array}{l}\text { - Message overhead is more due to implementation of thresholds } \\
\text { and Count Time. }\end{array}$ \\
\hline HEED [3] & $\begin{array}{l}\text { - } \quad \text { Provides a uniform } \mathrm{CH} \text { and load balancing. } \\
\text { - } \quad \text { Extends network lifetime by proper energy utilization. } \\
\text { iterations. }\end{array}$ & $\begin{array}{l}\text { Algorithm is suitable for specifically two-level hierarchical } \\
\text { networks. } \\
\text { Proper Connectivity is obtained only for a specified density } \\
\text { model and when a definite relationship exists between cluster } \\
\text { range and transmission range of a node. }\end{array}$ \\
\hline TL & - Less energy consumption than LEACH & - $\quad$ Assumes ideal homogenous network. \\
\hline $\begin{array}{l}\text { LEACH } \\
{[6]}\end{array}$ & $\begin{array}{l}\text { Two level clustering brings better energy load distribution } \\
\text { across the network }\end{array}$ & $\begin{array}{l}\text { - } \quad \text { Energy was not considered for } \mathrm{CH} \text { selection } \\
\text { - } \quad \text { Not suitable for large networks } \\
\text { - } \quad \text { Control overhead Complexity }\end{array}$ \\
\hline EECS [8] & $\begin{array}{l}\text { - } \quad \text { Low message overhead } \\
\text { - } \quad \text { More uniform distribution of clusters than LEACH }\end{array}$ & $\begin{array}{l}\text { - To calculate Cost metric, node must be aware of distance be- } \\
\text { tween adjacent } \mathrm{CH} \text { nodes and BS. } \\
\text { Single Hop communications limits the scalability of the algo- } \\
\text { rithm. }\end{array}$ \\
\hline EEUC [7] & $\begin{array}{l}\text { - Unequal clustering increases network lifetime. } \\
\text { - Relay node for Inter cluster communication are selected } \\
\text { based on cost, this reduces energy consumption. }\end{array}$ & $\begin{array}{l}\text { - Performing clustering in each round imposes large overheads } \\
\text { as all nodes participate for } \mathrm{CH} \text { Selection. } \\
\text { Residual energy was not considered for the Selection of relay } \\
\text { nodes, which can lead to new Hotspot problems. }\end{array}$ \\
\hline
\end{tabular}

\section{Conclusion}

Routing is rapidly increasing research area in WSN, owing to increase in size of WSN and its applications. The prerequisite for any routing algorithm in current scenario is energy efficiency, scalability and increased network lifetime. To tackle with large size networks distributed algorithm are required, as centralized algorithm are not scalable enough. In this paper, we have performed a comparative study of some prominent distributed cluster based routing algorithm. Each algorithm has its own strength and weaknesses. Future work involves designing and implementing a new clustering based routing algorithm.

\section{References}

[1] Xuxun Liu, A Survey on Clustering Routing Protocols in Wireless Sensor Networks", Sensors 2012, pp 11113-11153; https://doi.org/10.3390/s120811113.

[2] Heinzelman, W.R.; Chandrakasan, A.; Balakrishnan, H. Energy Efficient Communication Protocol for Wireless Microsensor Networks. In Proceedings of the 33rd Annual Hawaii International Conference on System Sciences, Maui, HI, USA, 4-7 January 2000; pp. 10-19. https://doi.org/10.1109/HICSS.2000.926982.

[3] O. Younis, S. Fahmy, "HEED: A Hybrid, Energy-Efficient, Distributed clustering approach for Ad Hoc sensor networks," IEEE Transactions on Mobile Computing, vol. 3, no. 4, pp. 366-379, 2004. https://doi.org/10.1109/TMC.2004.41.

[4] A. Manjeshwar and D. P. Agarwal, "TEEN: a routing protocol for enhanced efficiency in wireless sensor networks," In 1st International Workshop on Parallel and Distributed Computing Issues in Wireless Networks and Mobile Computing, April 2001. https://doi.org/10.1109/IPDPS.2001.925197.

[5] A. Manjeshwar and D. P. Agarwal, "APTEEN: A hybrid protocol for efficient routing and comprehensive information retrieval in wireless sensor networks," Parallel and Distributed Processing Symposium., Proceedings International, IPDPS 2002, pp. 195-202. https://doi.org/10.1109/IPDPS.2002.1016600.

[6] V. Loscri, G. Morabito and S. Marano, "A two-level hierarchy for low-energy adaptive clustering hierarchy (TL-LEACH)," VTC2005-Fall. 2005 IEEE 62nd Vehicular Technology Conference, 2005 2 2005, pp. 1809-1813. https://doi.org/10.1109/VETECF.2005.1558418.
[7] Li, C.F.; Ye, M.; Chen, G.H.; Wu, J. An Energy-Efficient Unequal Clustering Mechanism for Wireless Sensor Networks. In Proceedings of the 2nd IEEE International Conference on Mobile Ad-hoc and Sensor Systems Conference (MASS), Washington, DC, 7-10 November 2005; pp. 596-604.

[8] D. Wei, Y. Jin, S.Vural, K.Moessner\& R. Tafazolli, (2011) "An Energy-Efficient clustering solution for wireless sensor networks", IEEE transactions on wireless communications, Vol. 10, no. 11, pp.3973-3983. https://doi.org/10.1109/TWC.2011.092011.110717. 